Revue Française de Civilisation Britannique

\title{
La pratique référendaire en Écosse avant 2014
}

Referendums in Scotland before 2014

Jacques Leruez

\section{OpenEdition}

Journals

Édition électronique

URL : http://journals.openedition.org/rfcb/376

DOI : $10.4000 /$ rfcb.376

ISSN : 2429-4373

Éditeur

CRECIB - Centre de recherche et d'études en civilisation britannique

Référence électronique

Jacques Leruez, «La pratique référendaire en Écosse avant 2014 », Revue Française de Civilisation Britannique [En ligne], XX-2 | 2015, mis en ligne le 23 juillet 2015, consulté le 30 avril 2019. URL : http:// journals.openedition.org/rfcb/376; DOI : 10.4000/rfcb.376

Ce document a été généré automatiquement le 30 avril 2019

\section{(c) $(1) \odot$}

Revue française de civilisation britannique est mis à disposition selon les termes de la licence Creative Commons Attribution - Pas d'Utilisation Commerciale - Pas de Modification 4.0 International. 


\title{
La pratique référendaire en Écosse avant 2014
}

\author{
Referendums in Scotland before 2014
}

Jacques Leruez

1 Le recours au référendum au Royaume-Uni est intimement lié à l'adhésion à la Communauté européenne, même si l'on peut mentionner, en 1973, le «scrutin sur la frontière » (border poll) organisé par le Gouvernement Heath, en Irlande du Nord, bien qu'on ait, à son sujet, soigneusement évité de parler de référendum ${ }^{1}$. Les dirigeants britanniques sont bien conscients alors que ce vote ne résoudra pas le conflit; il s'agit avant tout, à leurs yeux, de mesurer le rapport des forces et même, plus simplement, de démontrer qu'un scrutin correct peut encore se dérouler dans la province, malgré la quasi guerre civile.

2 La situation politique à Londres en 1974-75 se caractérise par une grande instabilité due à des élections non décisives, en février et octobre 1974, ce qui génère des gouvernements faibles, incertains de leur avenir. Si l'élection de février permet à Harold Wilson de redevenir premier ministre ${ }^{2}$, c'est pour présider un gouvernement minoritaire qui ne retrouvera une majorité absolue (de trois voix à peine) qu'en octobre, et diriger un pays traumatisé par les affrontements sociaux. Ce pays a adhéré officiellement à la Communauté européenne, en janvier 1973, mais reste profondément divisé à propos de cette adhésion, non pas sur les lignes partisanes traditionnelles mais à l'intérieur même des deux grands partis et de leur électorat. D'où l'idée d'un recours au référendum.

3 Toutefois, cet instrument de démocratie directe est dénoncé, quelquefois violemment, à droite comme à gauche de la scène politique, dans la mesure où, en diverses époques et en divers lieux, il a servi à installer ou à fortifier des régimes autoritaires, voire dictatoriaux, mais aussi et surtout parce qu'il contrevient au principe fondamental qui régit le droit constitutionnel britannique, selon lequel "la Reine gouverne en son Parlement", principe qui prône la suprématie du Parlement et du Gouvernement qui en émane.

4 Ce principe a deux conséquences principales. D’une part, la volonté du Parlement s'impose sans limites, notamment celle de la Chambre des Communes, seule institution 
«nationale » élue au suffrage universel et censée refléter la volonté populaire. D’autre part, il n'y a pas de hiérarchie des textes en droit constitutionnel britannique, ce qui veut dire qu'aucune loi n'est juridiquement plus importante que les autres et est, par conséquent, discutée et adoptée selon une procédure similaire. Seuls les temps consacrés à chaque étape de la procédure peuvent varier.

5 Aussi, si le recours au référendum a fini par s'imposer, c'est pour deux raisons au moins. D'abord, parce que les partisans de l'adhésion savaient bien que, malgré le vote solennel et historique des Communes, le 28 octobre 1971 (356 voix pour, 244 voix contre), ce vote ne reflétait qu'imparfaitement la volonté de la population qui, d'après les sondages, était alors majoritairement hostile à l'adhésion. De plus, ce vote n'avait été acquis que grâce à la célèbre rébellion de 69 députés travaillistes, qui avaient approuvé le traité contre les consignes de leur parti, alors que 39 députés conservateurs votaient contre. Ensuite, précisément, comme ce vote l'indiquait, parce que le parti travailliste surtout était profondément divisé sur l'Europe, notamment en raison de la vive hostilité de la plupart des grands syndicats, dont le leader du parti devait tenir compte en raison, comme c'était le cas alors, du poids du vote syndical dans les congrès.

6 Toutefois, si Heath n'avait pas perdu l'élection du 28 février 1974, le parti travailliste aurait sans doute pu noyer ses divisions dans l'opposition. Ce ne fut pas le cas et Wilson, redevenu premier ministre, savait bien que la question européenne risquait fort de faire éclater son parti et son Gouvernement. D'où l'idée d'une "renégociation », clairement annoncée dans le manifeste électoral travailliste d'octobre 1974 et dont le principe fut accepté par les partenaires européens de la Grande-Bretagne. On sait que cette renégociation fut largement cosmétique; en tout cas, elle ne suffit pas à convaincre une majorité du parti travailliste de se rallier à l'adhésion, puisque, si la renégociation fut largement approuvée par les Communes, le 9 avril 1975, par 396 voix contre 170, c'est surtout grâce à l'appui de l'opposition. En effet, seuls 137 travaillistes votèrent pour, et 145 contre.

7 Wilson ne pouvait donc pas en rester là, d'où une seconde étape : le retour devant les électeurs comme seule issue possible, d'autant que l'opinion commençait à bouger en faveur de l'adhésion. Mais, comme il était difficile de provoquer une troisième élection en un peu plus d'un an, comme il l'avait envisagé tout d'abord, il opta pour le référendum. Ce référendum était officiellement consultatif, autorisé par le Parlement, qui juridiquement n'était pas lié par le résultat. Mais, comme les électeurs approuvèrent largement l'adhésion, il n'y eut pas conflit entre le politique et le juridique. Toutefois, si le résultat avait été inverse, on peut se demander si le Parlement se serait résigné à se déjuger, bien que Wilson eût déclaré qu'il s'inclinerait devant la volonté des électeurs. On serait peut-être allé alors vers une grave crise constitutionnelle.

Il reste, cependant, que le recours au référendum ne correspondait nullement à une volonté des dirigeants travaillistes de promouvoir un changement profond dans les règles de fonctionnement du système politique britannique mais, plus prosaïquement, au désir de trouver un processus, voire un subterfuge, pour dépasser les querelles de leur parti sur l'Europe et tenter d'éviter une scission qui, de toute façon, allait finir par se produire sept ans plus tard.

Les référendums en Écosse : résultats globaux

\begin{tabular}{|l|l|l|l|l|l|}
\hline Année & inscrits & votants & & Oui & Non \\
\hline
\end{tabular}




\begin{tabular}{|l|l|l|l|l|l|}
\hline 1975 & 3688799 & $61,7 \%$ & & $58,4 \%$ & $41,6 \%$ \\
\hline 1979 & 3747112 & $63,7 \%$ & & $51,6 \%$ & $48,4 \%$ \\
\hline \multirow{2}{*}{1997} & 3973673 & $60,4 \%$ & Q1 & $74,3 \%$ & $44,8 \%$ \\
\cline { 4 - 6 } & & & Q2 & $63,5 \%$ & $38,4 \%$ \\
\hline 2014 & 4283392 & $84,6 \%$ & & $44,7 \%$ & $55,3 \%$ \\
\hline
\end{tabular}

Questions posées :

- 1975: Pensez-vous que la Grande-Bretagne doit rester membre de la Communauté européenne?

- 1979 : Désirez-vous que les dispositions du Scotland Act 1978 soient mises en œuvre?

- 1997 : Q1 : Êtes-vous d'accord pour que soit créé un Parlement écossais?

- 1997 Q2 : Êtes-vous d'accord pour que ce Parlement dispose de pouvoirs fiscaux ?

- 2014 : L'Écosse doit-elle être indépendante ?

\section{Le référendum européen du 5 juin 1975}

9 Ce référendum ayant eu lieu dans un cadre britannique, l'Écosse y fut consultée comme simple partie du Royaume-Uni. Toutefois, du point de vue strictement écossais, le résultat ne fut pas sans signification. D'abord, la participation y fut plus faible qu'ailleurs : 61,7 \% au lieu de $64,8 \%$ en Angleterre et $66,7 \%$ au pays de Galles, ce qui traduisait un embarras, voire un certain manque d'enthousiasme. Ensuite, le Oui y fut moins ample qu'ailleurs : $58,4 \%$ contre $68,7 \%$ en Angleterre et $64,8 \%$ au pays de Galles. C'est que le parti travailliste y était encore plus divisé qu'ailleurs, mais c'était aussi et surtout parce que le Parti nationaliste - qui avait obtenu $30,4 \%$ des voix et 11 sièges aux Communes aux élections générales d'octobre 1974 - avait fait campagne pour le Non. D'ailleurs, Wilson redoutait un Non de l'Écosse, qui eût fait mauvais effet pour l'unité du royaume, et il avait envisagé de faire procéder à un dépouillement unique, centralisé à Londres, pour que n'apparaissent pas les différences régionales, mais il en avait été dissuadé par son administration, en raison des difficultés pratiques d'un tel arrangement.

Malgré tout, le Oui écossais était suffisamment franc (seules les Shetlands et les Western Isles avaient voté Non en majorité), à défaut d'être massif, pour apparaître comme une victoire pour le Gouvernement Wilson et, finalement, un revers pour le SNP. Plus généralement, ce vote montrait que la Grande-Bretagne était plus homogène et unie qu'on ne le pensait et que la prétention des anti-européens - dont faisait alors partie le SNP - à parler au nom du peuple britannique était pour le moins excessive.

\section{Le référendum du $1^{\mathrm{er}}$ mars 1979}

11 Propres à l'Écosse (et au pays de Galles), les référendums du $1^{\mathrm{er}}$ mars 1979 portaient sur la législation, dite de dévolution (Scotland Act et Wales Act 1978), que le Gouvernement Callaghan (qui avait succédé à Wilson en mars 1976) avait fait accepter péniblement par le Parlement, malgré les réserves de maints députés travaillistes, dont plusieurs travaillistes écossais, notamment le député de West Lothian, Tam Dalyell, auteur de la question restée 
célèbre (la West Lothian question), qui, jusqu'à nos jours, n'a pas reçu de réponse satisfaisante. d'arbitrages partisans au sein de la Chambre des Communes, qui tenaient peu compte de ce qui pouvait être bon pour l'Écosse, se résignèrent à faire campagne pour le Oui, tout en sachant fort bien la réticence, voire l'hostilité, de nombre de leurs partisans vis à vis d'un texte qui avait été conçu délibérément pour les tenir le plus longtemps possible loin du pouvoir. Si bien que le caractère ambigu de la réponse des électeurs, si elle signifiait avant tout une défaite pour le Gouvernement en place, était aussi un revers pour le SNP puisque, pour la seconde fois en quatre ans, il se trouvait dans le camp des perdants. Bien plus, en s'alliant, un mois plus tard (le 28 mars), aux conservateurs et aux autres petits partis pour voter une motion de défiance contre un gouvernement, certes déconsidéré et minoritaire, il hâta la venue au pouvoir de Margaret Thatcher et d'un gouvernement conservateur, qui allaient s'empresser de faire abroger le Scotland Act et, à la suite, de diriger l'écosse sans tenir compte de ses aspirations et de ses intérêts. Les électeurs écossais ne comprirent pas une telle attitude. Si le parti travailliste écossais ne souffrit pas, à l'élection suivante, de l'échec du référendum (il gagna même trois sièges : 44 sur 71 sièges écossais), le SNP, lui, perdit plus de 300000 voix et se retrouva avec deux sièges. Il commençait ainsi une longue traversée du désert.

\section{Le référendum du 11 septembre 1997}

Comme son prédécesseur de 1979, le référendum de septembre 1997 portait sur la question de la dévolution de pouvoirs à l'Écosse, mais plus explicitement que le 
précédent, sur la création (et pour certains la re-création après près de trois siècles) d'un Parlement bénéficiant d'une autonomie assez large.

Néanmoins, les circonstances politiques étaient entièrement différentes. La dévolution était proposée non pas par un gouvernement déconsidéré et minoritaire mais par une nouvelle équipe auréolée d'une des plus belles victoires du parti travailliste, y compris dans la seule Angleterre ; ce qui voulait dire, entre autres choses, qu'il ne dépendait pas, pour la mise en œuvre de son programme, des élus écossais et gallois, ce qui n'était pas arrivé souvent dans le passé. Ensuite, le livre blanc qui était soumis au vote des Écossais n'émanait pas seulement du manifeste électoral du parti travailliste; il avait été largement préparé en Écosse par les fonctionnaires du Scottish Office à partir des travaux des associations et conventions, certes non officielles mais néanmoins représentatives, travaux qui avaient abouti, en 1988, au Claim of Right for Scotland et aux rapports de la Scottish Constitutional Convention (en 1990 et 1995). Cette assemblée, les conservateurs et les nationalistes ayant refusé d'y prendre part pour des raisons opposées - les premiers refusant toute dévolution, les seconds parce que l'option «indépendance » n'était pas prise en compte - avait surtout regroupé des élus nationaux ou locaux, travaillistes et libéraux-démocrates, ainsi que des représentants de la société civile : syndicats, églises, associations diverses. Enfin, tous les députés écossais élus en mai 1997 étaient officiellement favorables à la dévolution, puisque tous les élus conservateurs de 1992 (onze) avaient été éliminés. D'où l'expression de « Tory-free Scotland ».

Alors, devant cette quasi-unanimité apparente, pourquoi organiser un référendum consultatif pré-législatif ? C'est d'abord que le nouveau premier ministre, Tony Blair, voulait aller vite : il avait promis de respecter ce qu'il appelait «John Smith's legacy » mais il ne voulait pas que la mise en œuvre de cet héritage retarde trop le programme du New Labour, d'autant qu'il avait en tête d'autres réformes institutionnelles qui nécessiteraient d'autres référendums. Il commença donc par le plus facile.

Blair savait ensuite que certains travaillistes écossais, parmi ceux qui avaient combattu pied à pied la loi de 1978, étaient toujours là. En organisant un référendum pré-législatif, il leur forçait la main. De fait, Tam Dalyell, le grand opposant de 1978, se manifesta assez peu et la discussion parlementaire prit le minimum de temps possible.

Restait enfin la question de la fiscalité. Les députés travaillistes écossais étaient - et le sont toujours - très sensibles à cette question, car ils savent bien que plus on donnera d'autonomie fiscale à l'Écosse, moins leur présence à la Chambre des Communes sera justifiée. Par conséquent, en posant la $Q 2$ du référendum, qui fut mal reçue par les médias écossais, car ils y virent une subtile manœuvre pour faire capoter l'ensemble du projet, Blair prenait un risque, mais finalement un risque calculé, d'abord parce que le pouvoir fiscal proposé était bien limité - la possibilité de faire varier de plus ou moins $3 \%$ les taux de l'impôt sur le revenu, et malgré tout, aucun des Parlements écossais qui se sont succédés depuis 1997 n'en a fait usage -, ensuite, parce que le nouveau gouvernement jouissait d'une telle marge de popularité en Écosse que les appréhensions bien réelles des classes moyennes et des milieux d'affaires furent facilement surmontées, la Q2 obtenant presque deux tiers de Oui (alors que la Q1 en obtenait presque les trois quarts). Toutefois, on ne peut parler d'une approbation pleine et entière de l'Écosse, compte tenu de l'importance des abstentions (près de $40 \%$ ).

21 À la différence des deux premiers référendums qui consistaient en l'approbation ou non d'une législation préexistante, votée dans les règles par le Parlement de Westminster et 
ayant déjà reçu la sanction royale, voire même un début d'application (c'était le cas pour l'adhésion à la Communauté européenne), le référendum de 1997 a été pré-législatif, la population écossaise n'ayant à se déterminer que sur un projet, certes détaillé et qui sera peu modifié par la législation définitive. Cela montre que le New Labour, malgré son succès électoral, avait gardé en mémoire les déboires du gouvernement Callaghan qui, à l'issue d'une bataille parlementaire qui avait duré près de deux ans, avait échoué à faire adopter un nouveau statut pour l'Écosse.

\section{Conclusion}

Il faut noter, en conclusion, que ce dernier référendum - comme tous ceux qui ont eu lieu avant ou après au Royaume-Uni - s'est tenu avec l'aval du Parlement de Westminster. La pratique britannique du référendum, qui fait maintenant partie de la coutume constitutionnelle, grâce aux précédents de 1975 et 1979, est donc très différente de la pratique française de la Ve République, notamment des référendums du président De Gaulle, qui étaient des scrutins de combat destinés à contourner, voire même à contrecarrer, la volonté parlementaire.

Enfin, le référendum de 1997, notamment parce que les institutions proposées (par exemple la création d'un vrai Parlement, pouvant librement voter la loi dans les domaines qui lui sont concédés, et non d'une simple assemblée de type régional) reprenaient largement les propositions faites par la convention écossaise, avait, dans une certaine mesure, la caractéristique d'un référendum d'auto-détermination, même si, juridiquement, le Scotland Act de 1998 fut, en définitive, l'œuvre de Westminster. Toutefois, si on était passé d'une charte octroyée (1978) à une charte discutée et élaborée en partie en Écosse (1997), seul le référendum de 2014 entrait pleinement dans la catégorie des référendums " permettant aux peuples de disposer d'eux-mêmes ", selon la définition de la Société des Nations. D'ailleurs, les électeurs écossais ne s'y sont pas trompés. En votant massivement en 2014, alors que les trois premiers référendums n'avaient suscité qu'une participation médiocre, ils montraient qu'ils avaient bien conscience que l'avenir de l'Écosse était vraiment en jeu.

\section{NOTES}

1. Le scrutin sur la frontière du 8 mars 1973 en Irlande du Nord posa deux questions : 1- Désirezvous que l'Irlande du Nord continue de faire partie du Royaume-Uni ? et 2- Désirez-vous que l'Irlande du Nord soit rattachée à la République d'Irlande à l'extérieur du Royaume-Uni ?. Sur 1 030084 électeurs inscrits, il y eut 604256 votants (58,5\%). Les partis et associations catholiques ainsi que le gouvernement de Dublin avaient appelé à un boycott du scrutin. Il y eut 591820 votes Oui à la première question (57,4 \% des inscrits) et 6463 votes Oui à la deuxième question $(0,6 \%$ des inscrits). Il y eut 5973 bulletins nuls (0,5\% des inscrits).

2. Il l'a déjà été d'octobre 1964 à juin 1970. 
3. John Smith (1938-1994) fut un député écossais, le prédécesseur de Blair à la tête du parti travailliste, et une personnalité éminemment respectée dans tous les milieux politiques. Chaud partisan d'une autonomie réelle pour son pays, il avait dirigé avec brio et courage, pour le gouvernement Callaghan, les débats qui avaient mené au vote du Scotland Act 1978. Il décéda prématurément avant d'avoir eu une chance d'accéder au poste de premier ministre.

\section{RÉSUMÉS}

Le référendum de 2014 était le quatrième référendum organisé en Écosse depuis que ce mode de consultation populaire a été introduit en Grande-Bretagne. Dans les deux premiers cas (1975, 1979), il s'agissait de la validation de lois déjà votées par le Parlement, mais qui avaient profondément divisé le parti au pouvoir ; le troisième (1997) consista en une consultation prélégislative à propos d'un projet détaillé largement inspiré par la convention constitutionnelle écossaise, organisme non élu mais néanmoins représentatif de la population. On peut donc estimer que ce référendum était d'une nature différente des deux premiers et se rapprochait d'une consultation, type Société des Nations, selon laquelle une population donnée était interrogée sur son avenir. Ce fut bien entendu totalement le cas en 2014.

The 2014 referendum was the fourth referendum organized in Scotland since this type of popular consultation was introduced in Britain. The object of the first two polls $(1975,1979)$ was to seek popular acceptance for two Acts of Parliament that had been passed in spite of deep divisions in the party in office. The third referendum was a pre-legislative poll on a detailed project inspired by the Scottish Constitutional Convention which, though non- elected, was widely representative of Scottish opinion. It can then be said that the 1997 referendum was of a different nature from the first two and more like the Society of Nations plebiscites by which a given population was asked to determine its future. It was of course still more so with the 2014 referendum.

\section{INDEX}

Mots-clés : Écosse, 5 juin 1975, 1er mars 1979, 11 septembre 1997, référendum sur l'indépendance

Keywords : Scotland, 5 June 1975, 1st March 1979, 11 September 1997, referendum

\section{AUTEUR}

\section{JACQUES LERUEZ}

Directeur de recherches honoraire au CNRS, président de l'Association Franco-Écossaise 